14. Kurtz, A., and Wagner, C. 1999. Cellular control of renin secretion. J. Exp. Biol. 202:219-225.

15. Nguyen, M., et al. 2002. Receptors and signaling mechanisms required for prostaglandin E2-mediated regulation of mast cell degranulation and IL- 6 production. J. Immunol. 169:4586-4593.

16. Tilley, S.L., et al. 2003. Identification of A3 receptor- and mast cell-dependent and -independent components of adenosine-mediated airway responsiveness in mice. J. Immunol. 171:331-337.

17. Juillerat, L., et al. 1990. Determinants of angioten$\sin$ II generation during converting enzyme inhibition. Hypertension. 16:564-572.

18. Urata, H., Kinoshita, A., Misono, K.S., Bumpus,
F.M., and Husain, A. 1990. Identification of a highly specific chymase as the major angiotensin IIforming enzyme in the human heart. J. Biol. Chem. 265:22348-22357.

19. Jenne, D.E., and Tschopp, J. 1991. Angiotensin II-forming heart chymase is a mast-cell-specific enzyme. Biochem. J. 276:567-568.

20. Nataraj, C., et al. 1999. Angiotensin II regulates cellular immune responses through a calcineurindependent pathway. J. Clin. Invest. 104:1693-1701.

21. Theoharides, T.C., and Cochrane, D.E. 2004. Critical role of mast cells in inflammatory diseases and the effect of acute stress. J. Neuroimmunol. 146:1-12.

22. Constantinides, P. 1995. Infiltrates of activated mast cells at the site of coronary atheromatous erosion or rupture in myocardial infarction [editorial]. Circulation. 92:1083.

23. Kovanen, P.T., Kaartinen, M., and Paavonen, T. 1995. Infiltrates of activated mast cells at the site of coronary atheromatous erosion or rupture in myocardial infarction. Circulation. 92:1084-1088.

24. Laine, P., et al. 1999. Association between myocardial infarction and the mast cells in the adventitia of the infarct-related coronary artery. Circulation. 99:361-369.

25. Hara, M., et al. 2002. Evidence for a role of mast cells in the evolution to congestive heart failure. J. Exp. Med. 195:375-381.

\title{
Pathomechanisms in rheumatoid arthritis - time for a string theory?
}

\author{
Cornelia M. Weyand and Jörg J. Goronzy
}

Kathleen B. and Mason I. Lowance Center for Human Immunology, Department of Medicine, Emory School of Medicine, Atlanta, Georgia, USA.

\begin{abstract}
RA is a quintessential autoimmune disease with a growing number of cells, mediators, and pathways implicated in this tissue-injurious inflammation. Now Kuhn and colleagues have provided convincing evidence that autoantibodies reacting with citrullinated proteins, known for their sensitivity and specificity as biomarkers in RA, enhance tissue damage in collagen-induced arthritis (see the related article beginning on page 961). This study adds yet another soldier to the growing army of autoaggressive mechanisms that underlie RA. With great success researchers have dismantled the pathogenic subunits of RA, adding gene to gene, molecule to molecule, and pathway to pathway in an ever more complex scheme of dysfunction. The complexity of the emerging disease model leaves us speechless. It seems that with this wealth of data available, we need to develop a new theory for this disease. We may want to seek guidance from our colleagues in physics and mathematics who have successfully integrated their knowledge of elementary particles and the complexity of their interacting forces by formulating the string theory.
\end{abstract}

Most scientists agree that simplicity is beautiful, alluding to the fact that true understanding of a natural phenomenon is reached when it can be expressed as a simple formula. Albert Einstein led the physicists in the ultimate quest for a theory that would provide a comprehensive description of the laws of nature. Witnessing the growing complexity of physics, Einstein believed that there was a need for a "unified field theory" and was convinced that it would bring the beauty of general relativity to all of nature's laws.

Einstein did not succeed, but in the last 3 decades a theory called string theory has gained

Nonstandard abbreviations used: anti-CCP, anticyclic citrullinated peptide; CII, bovine type II collagen.

Conflict of interest: The authors have declared that no conflict of interest exists.

Citation for this article: J. Clin. Invest. 116:869-871 (2006). doi:10.1172/JCI28300. momentum, promising that it can provide a unified theory of all the elementary particles and their interactions incorporating Einsteinian gravitation and other fields (1). If physicists are coming closer to obtaining a theory that describes all laws of nature, does that raise hope for us that an understanding of

\section{Horror autotoxicus - autoantibodies in RA}

The first immune abnormality described in patients with RA was the production of autoantibodies, so-called "rheumatoid factors," directed against the constant region of $\operatorname{IgG}(2)$. Fifty years ago it seemed reasonable to hypothesize that the patients' dilemma was indeed a scenario of horror autotoxicus. Rheumatoid factors became critically important as diagnostic tools, but why they were generated and how precisely such a complex puzzle as RA is within reach? they participated in synovitis remained less well defined. The question of whether rheumatoid factors are pathogenic or are instead an epiphenomenon of chronic autoimmune disease has plagued the field of rheumatology for several decades.

The description of antibodies to citrullinated proteins, first identified as anti-perinuclear factor and anti-keratin antibodies, has revived some of these discussions $(3,4)$. Anti-cyclic citrullinated peptide (anti-CCP) antibodies appear to be rather sensitive $(68 \%$ sensitivity) and highly specific (98\% specificity) for RA (5). These autoantibodies were first seen as binding reactivity to filaggrin, a protein that is not expressed in the synovium and is typically found during terminal differentiation of epithelial cells. However, other proteins, such as fibrin, that have undergone posttranslational citrullination could serve as a target in the joint and thus represent the arthritogenic antigen recognized by pathogenic autoantibodies.

\section{Anti-CCP antibodies - a disease amplifier}

In an elegant study published in the current issue of the JCI, Kuhn and colleagues (6) have examined the pathogenic role of anti-CCP antibodies in an animal model of autoimmune arthritis. Arthritis was induced in mice by immunization with bovine type II collagen (CII). Immunization not only led to the production of anti-CII antibodies but also induced anti-CCP antibodies. Interestingly, both types of autoantibodies could be detected prior to frank 


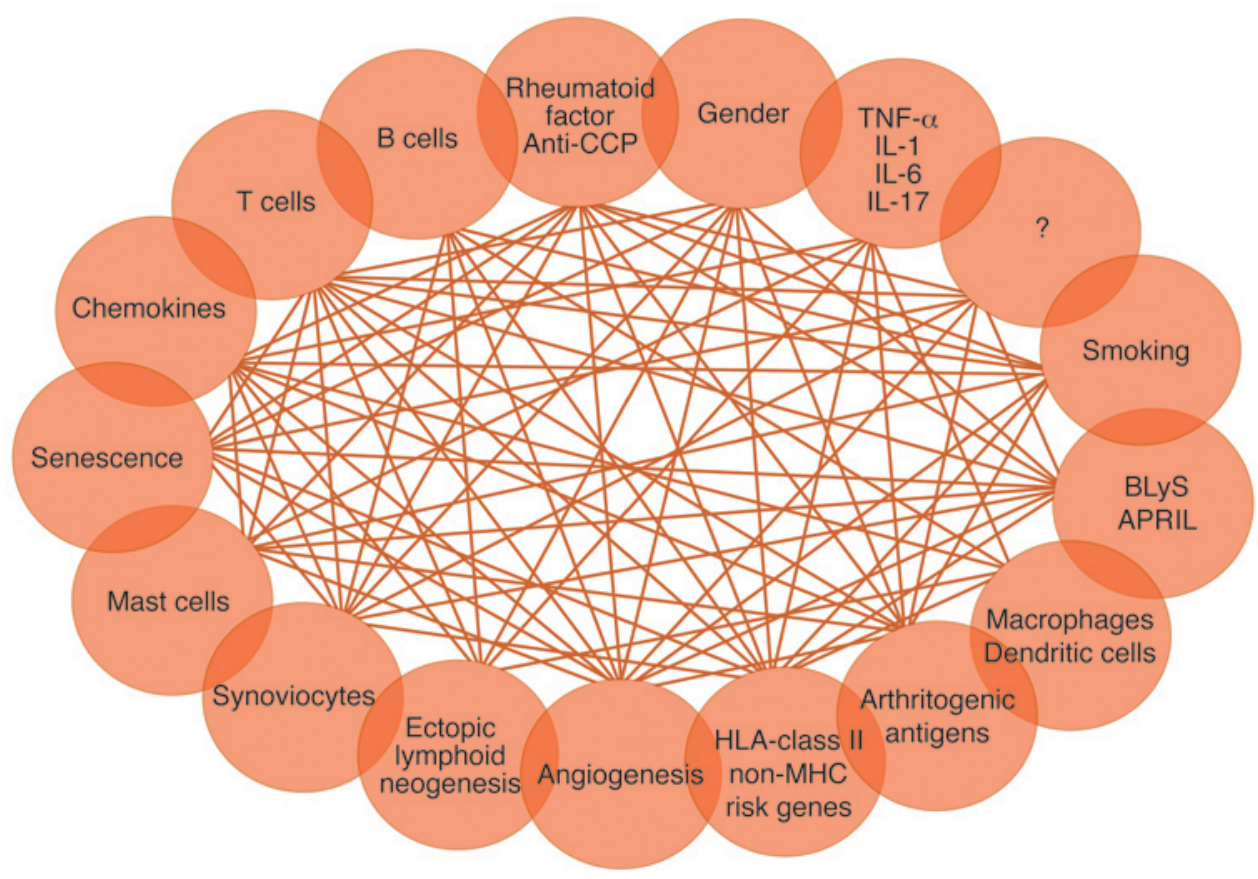

Figure 1

The complexities of RA. This scheme outlines the multiplicity of pathogenic factors and the intricacies of their interactions in causing breakdown of self tolerance and tissue-destructive inflammation. APRIL, a proliferation-inducing ligand; BLyS, B lymphocyte stimulator.

joint inflammation. When mice were tolerized with a citrulline-containing peptide, immunization with CII caused reduced disease severity and incidence. Also, antibodies to citrullinated fibrinogen enhanced arthritis when coadministered with antiCII antibodies. The authors concluded that anti-CCP antibodies are not simply an epiphenomenon of chronic inflammation but are directly involved in tissue injury.

The authors deserve praise for their study design (6), which settles the question of whether anti-CCP antibodies are merely a part of chronic immune stimulation or participate in tissue damage. Reduced disease severity in animals tolerized against citrullinated peptides clearly assigns a role to these autoantibodies as disease enhancers. Experiments in which monoclonal antibodies reactive to $\mathrm{CCP}$ were transferred into mice emphasize that anti-CCP alone cannot induce arthritis but needs to be combined with a cocktail of antibodies to CII. The study does not provide a mechanism through which anti-CCP antibodies boost synovitis, but transfer of anti-CCP antibodies demonstrated binding in the target tissue. Notably, anti-CCP antibodies bound only in the inflamed pannus but not in normal synovial tissue. In summary, autoantibodies with different specificities seem to be cooperating in enhancing inflammatory damage to the synovial membrane. In isolation, anti-CCP seems not to have arthritogenic potential, supporting the notion that anti-CCP antibodies need to be added to the growing number of pathomechanisms that act as disease amplifiers.

Looking proudly on the accomplishments of research in RA, the time may have come to step back and ask whether we have succeeded in collecting the necessary information to formulate a unified field theory for this disease. Are we ready for a theory that brings together the bits and pieces of our understanding of autoimmune arthritis?

\section{So many pathomechanisms in RA - a need to assemble the mosaic}

A glance at a scheme summarizing the growing knowledge of pathomechanisms in RA (7) easily communicates our dilemma (Figure 1). Fifty years ago a single mechanism, the unfortunate production of autoreactive rheumatoid factors, dominated the pathogenic model, but now we have complex diagrams connecting a multitude of cells through a vast number of arrows. Nobody would doubt that at least 6 different cell types, including synovial fibroblasts, macrophages, T cells, B cells, dendritic cells, and mast cells, all hold critical positions in sustaining synovitis. But does one of them stand out as the ultimate instigator? Nobody would doubt that cytokines are critically involved in amplifying inflammation and mediating tissue injury, but is there a master cytokine?

Highly complex pathways of tissue damage and repair are unquestionably involved in creating and maintaining the lesion that harms cartilage and bone. The pannus could not grow unless supported by a network of new blood vessels (8). Rheumatoid synovitis is closely associated with the process of lymphoid neogenesis (9), which provides a highly efficient microarchitecture for optimized immune recognition events (10). The lesion itself produces growth and survival factors for B cells (11), creating ideal conditions for their development and maturation. B cells living in the inflamed synovial membrane may release anti-CCP antibodies, thereby directly aggravating tissue injury. T cells recruited to the lesion encounter a microenvironment in which they receive stimulatory signals through legions of ligands expressed on nonlymphoid cells (12). All these processes are complex by themselves and, in the inflamed joint, they seem to coexist.

Teaching students and fellows about the pathogenesis of RA has become a challenging task. We have acquired so much knowledge about the molecular details of the different inflammatory cells and mediators, but what we are yearning for is a theory that would fit it all together, under 
one formula, one idea, one mechanism - as scientists from many other disciplines have already actively sought.

Of course, there is ample reason to believe that such a vision is not achievable, at least not in the foreseeable future. If indeed RA is an infectious disease, critical clues may be out of reach unless technology development enables us to overcome limitations in the recognition, identification, and characterization of infectious microorganisms.

Alternatively, autoimmune diseases may be just a mess, lacking a unifying disease concept and representing nothing more than an overwhelming conglomerate of defective cells, mediators, and pathways. Admittedly, pathogenic concepts of RA have not been very predictive of suitable molecular targets. Often, therapeutic successes have been brought forward by simply applying therapies used in other conditions to patients with RA (13). And experimental serendipity has indeed been quite successful. In hindsight, we may have to look for why and how these interventions work. By adding more culprits to the pathogenic scheme, we can find explanations for why B cell-targeted therapies (14) and blockade of $\mathrm{T}$ cell costimulation (15) provide therapeutic benefit.

\section{Bringing light to the jungle - a unified theory of autoimmunity}

How could we go about formulating a string theory of autoimmunity? Einstein had understanding of the central concepts of general relativity long before he sat down and developed the equations describing these laws of nature. String theory originated from the simple and beautiful bootstrap principle, named after the old tale about the man who could pull himself up by his own bootstraps (16). The theory then evolved over decades. The idea is that a set of particles can be treated as if it is composed of combinations of the same particles. It may sound like heresy to propose a theoretical approach to human disease, but it could offer insights that remain hidden from current experimental approaches.

String theory has been an important nucleus for experimentalists and has proven to be applicable to diverse fields (17) such as quark confinement, quantum mechanics of black holes, and pure geometry. One would thus predict that a string theory of RA would allow for generalization to other autoimmune diseases, generating unexpected ideas that may reach much further than even the immune system. It is reassuring that studies such as the one by Kuhn and colleagues (6) can be designed to test detailed aspects of how the immune system attacks joints and that experimental systems can be built to investigate whether a hypothesis is correct or not.

Big ideas, like a generalization of how the immune system fails and causes inflammatory destruction of tissues, come along rarely. Leaving behind the concept of horror autotoxicus, which is now almost 100 years old, and proposing a totally new view of autoimmunity could be a good start. Maybe the professional theorists can help. Theory building is at its best when a science has matured, when a large body of observations is available, and when the power of theoretical methods has grown (18). Considering the enormous progress that has been made in understanding what goes wrong in RA, the time may have come to step out of the boundaries of current paradigms and work to formulate theories that are able to synthesize the complexities of autoimmune disease.

\section{Acknowledgments}

The authors thank Sergey Pryshchep for preparing the figure and Tamela Yeargin for editing the manuscript. This work was funded in part by grants from the National Institutes of Health (RO1 AR 41974, RO1 AR 42527, and RO1 AI 44142).

Address correspondence to: Cornelia M. Weyand, Lowance Center for Human
Immunology, Emory University School of Medicine, Room 1003, Woodruff Memorial Research Building, 101 Woodruff Circle, Atlanta, Georgia 30322, USA. Phone: (404) 727-7310; Fax: (404) 727-7371; E-mail: cweyand@emory.edu.

1. Zwiebach, B. 2004. A first course in string theory. Cambridge University Press. Cambridge, Massachusetts, USA. 578 pp.

2. Kunkel, H.G., and Williams, R.C. 1964. Rheumatoid arthritis. Annu. Rev. Med. 15:37-52.

3. Nienhuis, R.L., and Mandema, E. 1964. A new serum factor in patients with rheumatoid arthritis; the antiperinuclear factor. Ann. Rheum. Dis. 23:302-305.

4. Simon, M., et al. 1993. The cytokeratin filament-aggregating protein filaggrin is the target of the so-called "antikeratin antibodies," autoantibodies specific for rheumatoid arthritis. J. Clin. Invest. 92:1387-1393.

5. Schellekens, G.A., et al. 2000. The diagnostic properties of rheumatoid arthritis antibodies recognizing a cyclic citrullinated peptide. Arthritis Rheum. 43:155-163.

6. Kuhn, K.A., et al. 2006. Antibodies against citrullinated proteins enhance tissue injury in experimental autoimmune arthritis. J. Clin. Invest. 116:961-973. doi:10.1172/JCI25422.

7. Goronzy, J.J., and Weyand, C.M. 2005. Rheumatoid arthritis. Immunol. Rev. 204:55-73.

8. Paleolog, E.M. 2002. Angiogenesis in rheumatoid arthritis. Arthritis Res. 4:S81-S90.

9. Takemura, S., et al. 2001. Lymphoid neogenesis in rheumatoid synovitis. J. Immunol. 167:1072-1080.

10. Kang, Y.M., et al. 2002. CD8 T cells are required for the formation of ectopic germinal centers in rheumatoid synovitis. J. Exp. Med. 195:1325-1336.

11. Seyler, T.M., et al. 2005. BLyS and APRIL in rheumatoid arthritis. J. Clin. Invest. 115:3083-3092. doi:10.1172/JCI25265.

12. Goronzy, J.J., and Weyand, C.M. 2004. T-cell regulation in rheumatoid arthritis. Curr. Opin. Rheumatol. 16:212-217.

13. Chen, Q., and Wei, W. 2005. New therapeutic approaches for rheumatoid arthritis. Assay Drug Dev. Technol. 3:329-337.

14. Edwards, J.C., et al. 2004. Efficacy of B-cell-targeted therapy with rituximab in patients with rheumatoid arthritis. N. Engl. J. Med. 350:2572-2581.

15. Kremer, J.M., et al. 2005. Treatment of rheumatoid arthritis with the selective costimulation modulator abatacept: twelve-month results of a phase iib, double-blind, randomized, placebo-controlled trial. Arthritis Rheum. 52:2263-2271.

16. Gell-Mann, M. 1994. The quark and the jaguar: adventures in the simple and the complex. W.H. Freeman and Sons. New York, New York, USA. 392 pp.

17. Witten, E. 2005. Unravelling string theory. Nature. 438:1085.

18. Gell-Mann, M. 2001. Consciousness, reduction, and emergence. Some remarks. Ann. N. Y. Acad. Sci. 929:41-49. 


\title{
Ontogeny of adrenal steroid biosynthesis: why girls will be girls
}

\author{
Perrin C. White \\ Department of Pediatrics, University of Texas Southwestern Medical Center, Dallas, Texas, USA.
}

\begin{abstract}
Male and female external genitalia appear identical early in gestation. Testosterone exposure at 8-12 weeks' gestation causes male differentiation. Female fetuses virilize if their adrenals secrete excessive levels of androgens, as occurs in congenital adrenal hyperplasia due to 21-hydroxylase deficiency. This can be ameliorated by administering dexamethasone to the mother. A study by Goto et al. in this issue of the JCI provides a rationale for this treatment by demonstrating that the fetal hypothalamic-pituitary-adrenal axis is fully functional when the genitalia differentiate (see the related article beginning on page 953). Dexamethasone suppresses this axis, reducing abnormal secretion of adrenal androgens. Their results also show that cortisol synthesis by the fetal adrenal decreases after this period, allowing the adrenal to secrete high levels of dehydroepiandrosterone, an androgen precursor. However, this does not virilize female fetuses because androgens are aromatized to estrogens in the placenta. Thus normal sexual differentiation requires exquisite timing of fetal cortisol and androgen secretion versus placental capacity for aromatization.
\end{abstract}

Early in gestation, the external genitalia are anatomically identical in both sexes. Female external genitalia represent the default state with male differentiation occurring if high levels of testosterone (as secreted by fetal testes) induce steroid $5 \alpha$-reductase type 2 (SRD5A2) and are then converted to dihydrotestosterone in genital skin. The critical period for such differentiation is $8-12$ weeks post conception (wpc). Later exposure to testosterone increases growth of the penis or clitoris, but does not induce fusion of labia majora and minora into scrotum and penile urethra, respectively. Differentiation of the genitalia can also be influenced by adrenal steroid biosynthesis. The early ontogeny of such biosynthesis has now been studied by Goto et al. (1) in this issue of the JCI (a preliminary report of some of these findings was published previously; see ref. 2).

\section{Endocrinology of human pregnancy}

To place this work in context, consider the endocrinology of human pregnancy (Figures

Nonstandard abbreviations used: CYP21, cytochrome P450 21-hydroxylase; DHEA, dehydroepiandrosterone; DHEAS, dehydroepiandrosterone sulfate; HPA, hypothalamic-pituitary-adrenal; HSD3B, $3 \beta$-hydroxysteroid dehydrogenase; wpc, weeks post conception.

Conflict of interest: The author has declared that no conflict of interest exists.

Citation for this article: J. Clin. Invest. 116:872-874 (2006). doi:10.1172/JCI28296.
1 and 2). During early gestation, the estradiol required to maintain pregnancy is provided by the corpus luteum in the maternal ovary. But after 8 weeks' gestation, most estradiol is synthesized by the fetoplacental unit (3). Dehydroepiandrosterone sulfate (DHEAS) is secreted by the fetal adrenal cortex. Synthesis of DHEAS requires importation of precursor cholesterol into mitochondria, regulated by the steroidogenic acute regulatory (StAR) protein, followed by conversions mediated by the cholesterol side-chain cleavage enzyme (CYP11A), 17 $\beta$-hydroxylase/17,20lyase (CYP17) and steroid sulfotransferase (SULT2A1). In the placenta, steroid sulfatase (also known as arylsulfatase, ARSC1) converts DHEAS back to dehydroepiandrosterone (DHEA), which is converted to androstenedione by $3 \beta$-hydroxysteroid dehydrogenase type 1 (HSD3B1; an isozyme distinct from the HSD3B2 expressed in the adrenal cortex). Androstenedione is aromatized to estrone by CYP19 and then converted to estradiol by HSD17B1.

Additionally, DHEAS is oxidized in the fetal liver to $16 \alpha-\mathrm{OH}$ DHEAS, which is converted by the placenta to estriol by the same enzymes that are involved in estradiol synthesis. Although estriol has little known functional significance, it does not circulate in the blood of nonpregnant women at high levels and is therefore a marker of function of the fetoplacental unit.
Thus DHEAS is the main steroid secreted by the fetal adrenal cortex during mid-gestation (Figure 2). Fetal cortisol is required later in pregnancy for lung maturation, particularly surfactant production, but this cannot be allowed to occur too early because amniotic fluid surfactant levels may control the timing of parturition (4). Maternal cortisol cannot normally reach the fetus because it is oxidized to cortisone, an inactive steroid, by HSD11B2 in the placenta (5). A low ratio of fetal cortisol to DHEAS secretion is maintained by decreased expression of adrenal HSD3B2, which is required for cortisol biosynthesis.

Placental aromatase prevents female fetuses (as well as their mothers) from virilization by the large amounts of DHEAS secreted by the fetal adrenal cortex. Conditions under which placental aromatase activity is decreased (including congenital deficiencies of aromatase or its accessory enzyme, P450 oxidoreductase; ref. 6), are associated with fetal and maternal virilization. Placental aromatase can also protect female fetuses from virilization when the mothers themselves have high circulating testosterone levels resulting from poorly controlled congenital adrenal hyperplasia due to CYP21 deficiency (7). This observation implies that high placental aromatase expression too early in pregnancy might interfere with normal male differentiation, but then how is the female fetus protected from virilization before placental aromatase expression has reached high levels?

To answer this question, Goto et al. (1) examined expression of the enzymes required for cortisol biosynthesis in fetuses at 7-10 wpc; previously, the youngest human fetuses studied were 14 weeks old (8). The authors found that adrenal explants from 8-wpc fetuses have a robust capacity to secrete cortisol, and that this secretion is responsive to adrenocorticotrophic hormone (ACTH). By $10 \mathrm{wpc}$, cortisol secretion begins to decrease. Thus they propose that cortisol secretion is relatively 